\title{
PERSONA JURÍDICA, ¿UNA FICCIÓN?
}

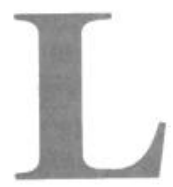

a cuestión de la conceptualización (la naturaleza) de una persona jurídica ha sido uno de los conjuntos de problemas más debatidos en el Derecho de sucesiones tanto en la Europa continental como en Finlandia. Además, se puede decir, sin riesgo de equivocarse, que la cuestión continúa, en cierto sentido, siendo un problema recurrente en la ciencia jurídica. Presumiblemente, esto se debe a que el problema está conectado de una forma particularmente interesante con la aproximación jurídica que domina en cada período. El motivo específico para la presentación que sigue ha sido la observación de que la reciente discusión filosófica ha producido puntos de vista que son significativos también para la ciencia jurídica, y en cierta medida estos puntos de vista dan nueva luz sobre las opiniones aceptadas. Me estoy refiriendo a la forma de pensar a la que se llama filosofía analítica, una escuela de pensamiento que también se ha asentado firmemente en los países nórdicos. Esta forma de pensar se usará más adelante para abordar el problema.

La considerable disparidad de opiniones sobre la persona jurídica ha creado un número de respuestas tan heterogéneas que, sin tener en cuenta la conceptualización que adoptemos como punto de partida, la discusión parece conducir inevitablemente a un punto muerto. A este respecto, apenas sería apropiado buscar argumentos en apoyo de cualesquiera de las anteriores teorías sobre la persona jurídica. Parece más beneficioso cambiar drásticamente el punto de vista inicial y presentar la cuestión como sigue: ¿cuál ha sido la razón de la división de opiniones que se ha mostrado tan improductiva? ¿Por qué, a pesar de todos los esfuerzos, no ha sido posible llegar a un acuerdo? Antes de intentar justificar mi propia respuesta a esta cuestión, quiero tratar brevemente algunas soluciones que se han dado con anterioridad al problema de la persona jurídica.

Esencialmente cuando se trata de fundaciones (y masas hereditarias) se podría posiblemente opinar que la totalidad del patrimonio formaría en sí misma un sujeto jurídicamente relevante. Un ejemplo de esto, que se ha mencionado, es la «hereditas iacens» en derecho romano, según la cual la propiedad (el patrimonio) de un causante, entre la muerte del causante y la aceptación de la herencia, es el sujeto de derechos y obligaciones que sobreviven al causante. La «hereditas iacens» ofrece un medio por el que se evitaría el (temporalmente) extraño concepto de propiedad sin propietario. De esta forma era posible entender, de forma natural, un fenómeno jurídico, la transferencia directa de derechos de un sujeto a otro.

En relación con esto, se ha hecho a veces referencia a la teoría del «Patrimonio con un fin específico», un concepto que alude a la posibilidad de que al patrimonio perteneciente a una persona jurídica le falte un titular. Los derechos y obligaciones sólo se mantienen unidos por el fin al que está afectado el patrimonio en su conjunto. Quizá no sea completamente incorrecto decir que esta última conceptualización es, en cierto sentido, un refi- 
namiento de la conceptualización de la «hereditas iacens». Sólo requiere que abandonemos la necesidad convencional de un sujeto y un objeto.

Según las llamadas teorías realistas de la persona jurídica, una entidad formada por diversas personas (accionistas, miembros, herederos, etc.) constituye una persona colectiva, un sujeto de derecho. Sin embargo, tal sujeto no es una persona natural -como se ha dicho- y sólo puede existir en el mundo jurídico. Es un sujeto artificial que se ha creado por personas con fines jurídicos. Lo esencial en esta idea, tal como yo la entiendo, es que supone la existencia (al menos en algún sentido) de un sujeto que no es persona física. Está claro, que es precisamente por esto por lo que se dice, por ejemplo, que los derechos y obligaciones no incumben a las personas físicas que crean la persona jurídica ni a los miembros de esta persona jurídica ni a sus administradores, sino que tales derechos y obligaciones incumben a un sistema jurídico supraindividual que puede separarse de las personas que lo componen.

Esta concepción muestra su formulación más clara cuando al sujeto de derecho en cuestión se le dan también características personales: se habla, por ejemplo, de la existencia y objeto de la persona jurídica, y se hace referencia a la organización como algo con voluntad propia. Con todo, últimamente se ha tratado de evitar tales expresiones en la medida de lo posible, o al menos se ha hecho hincapié en que sólo se trata de una forma alegórica de hablar. Así, aunque se asume la existencia de personas físicas, la atribución de personalidad a la persona jurídica se considera como una ficción. Por tanto; se podría llamar a tal consideración (una consideración que creo ha sido -al menos en fechas cercanas- bastante común en la ciencia jurídica finlandesa) una teoría de la ficción, en especial porque esta teoría, cuando se interpreta en la forma vista, se aproxima bastante a la teoría de Savigny que tiene este nombre.

Los intentos antes descritos de hallar una solución se han criticado, a veces, aludiendo a la inutilidad de la aproximación llamada sustancial. Y, esto se debe a que se ha considerado, al menos en muchos casos, que estas teorías describen una consideración típica de la naturaleza de los fenómenos basada en especulaciones metafísicas. Presumiblemente, algunas de las más destacadas opiniones tienden a adoptar una formulación linguística que refleja un tipo especial de perspectiva ontológica; con esto me refiero a la opinión de que, junto a las personas físicas, «existe» realmente (al menos en el mundo jurídico) algún tipo de personalidad individual jurídica. Con todo, debe recalcarse que tal opinión ha dado lugar últimamente a la opinión que he llamado la teoría de la ficción.

Para mayor claridad, el problema de la sustancia y su inutilidad puede, $\mathrm{y}$, tal como lo veo, debe reconducirse desde el plano matafísico al lógico. Además, en este caso, los intentos de construir una conceptualización pueden interpretarse sin dificultad, como intentos de obtener definiciones, que posibiliten el acuerdo sobre ciertos criterios en el uso del concepto de persona jurídica. En otras palabras, se ha intentado hallar las condiciones necesarias y suficientes para, cuando se dan ciertos hechos, poder hablar de «persona jurídica».

Por consiguiente, los intentos de construir una conceptualización han conducido, casi sin excepción, a una cierta unilateralidad. Pese a ingentes 
esfuerzos, no ha sido posible alcanzar un acuerdo en la doctrina sobre los requisitos conceptuales de la persona jurídica (su naturaleza). Esto es naturalmente una de las más importantes dificultades en las teorías, pero al mismo tiempo guarda relación con una característica que parece ser bastante interesante en relación con la solución del problema.

El hecho de que difieran las opiniones se debe presumiblemente en gran medida a que la cuestión que se ha tomado como punto de partida ( ${ }_{i}$ Es $\mathrm{X}$ una persona jurídica?») se ha formulado incorrectamente. En general, no puede ser contestada. Y, esto es así porque la cuestión conduce inevitablemente a una situación en la que cada autor trata de cristalizar la definición del concepto aún cuando los «fenómenos» que pertenecen al dominio del concepto (la expresión) no tengan una única característica común que pueda servir como criterio. Además, es incorrecto en principio intentar hallar una definición de persona jurídica universalmente aplicable. Por esta razón tampoco podemos presentar nuestro problema en la forma: «¿Cuál es la naturaleza de una persona jurídica (de una masa hereditaria)?»

La cuestión de la persona jurídica es un problema que tiene que ver con las reglas del lenguaje que usamos y por esta razón sólo puede resolverse examinando cuidadosamente estas reglas. Mi tesis principal -que en este momento ya se puede presentar de forma preliminar- es por tanto la siguiente: el significado del concepto de «persona jurídica» («sociedad», etc.) se manifiesta en la forma en que estos términos son y pueden ser usados en conexión con el lenguaje jurídico.

Pienso que éste que uno de los impulsos que condujo a Lahtinen a estudiar esta cuestión de una forma no convencional. Él mismo llamó semántico a su punto de vista. En lo que sigue trataré de analizar alguno de los elementos esenciales de este punto de vista. En los fenómenos jurídicos, se trata siempre del comportamiento humano. Por esto, se necesita un nombre para denominar jurídicamente a las personas jurídicas. En principio es posible arreglárselas usando sólo nombres de personas físicas. En algunos casos, sin embargo, esto puede ser bastante inapropiado. Éste es específicamente el caso de una comunidad bien organizada. Por esta razón, no son descritas con nombres de personas físicas sino con la ayuda en cada caso de un símbolo especial (una descripción; «Kennzeichung») para la organización. Lahtinen entendía la palabra «Kennzeichung» como un símbolo que, sin ser el nombre del fenómeno (organización) en cuestión, define sin ambigüedad este fenómeno. Con respecto a esto, Lahtinen hace referencia por ejemplo a las opiniones de Carnap.

El problema de la persona jurídica aparece en un estudio que usa este punto de partida como una cuestión de si se permite o no el «Kennzeichung». Según Lahtinen, ésta, a su vez, depende de las bases sobre las que el «Kennzeichung» puede ser traducido con suficiente certeza en nombres de personas físicas. En opinión de Lahtinen el uso del «Kennzeichung» requiere la existencia de una organización que permita tal traducción. Si este es el caso, podemos decir que la organización en cuestión es una persona jurídica. 
Desde este punto de vista, el significado del «Kennzeichung» es el mismo que el de los nombres de personas. Es un medio para redescubrir ciertas personas físicas. De acuerdo con esto, Lahtinen señala que no se da la distinción, que aparece en la concepción dominante, entre la persona jurídica y los individuos que la componen. El problema de la naturaleza de la persona jurídica, un problema que ha causado gran cantidad de discusión, desaparece. En su lugar, están las cuestiones de la organización técnica a las que merece la pena prestar atención.

En un examen más a fondo de la forma de pensar de Lahtinen, se puede notar que, a pesar de su claridad, deja unas cuantas cuestiones abiertas. En particular, no se han aclarado dos puntos. En mi opinión, estos dos puntos son esenciales para comprender su postura. En primer lugar, no está claro lo que en última instancia implica llamar a una cierta designación colectiva un «Kennzeichung». Nadie puede responder a esto, sin a su vez, estudiar antes lo que se puede entender por el proceso de «traducir» el «Kennzeichung» a nombres personales. En lógica de enunciados, ¿cómo es posible realizar el proceso de «traducción»?

Para ilustrarlo vamos a estudiar, por ejemplo, la proposición «La sociedad de responsabilidad limitada X posee el patrimonio r»

Parecidas proposiciones relativas a otras organizaciones son, entre todas, «La fundación $\mathrm{Y}$ posee el patrimonio $\mathrm{r} » \mathrm{y}$ «La masa hereditaria $\mathrm{Z}$ posee el patrimonio $\mathrm{r} »$.

Lahtinen señala, por ejemplo, que el «Kennzeichung» hace referencia a ciertas personas, y se pueden hallar estas personas «refiriéndose» al «Kennzeichung». No obstante, esto requiere algún conocimiento de la organización. Si el grado de conocimiento es suficientemente alto, se puede usar indistintamente el «Kennzeichung» o los nombres personales. Lahtinen presenta como ejemplo (un ejemplo, que en comparación con el problema completo, está supersimplificado) el «Kennzeichung» «los bienes comunales en el Lago X». Usando ciertos hechos, tales como las normas aplicables y la lista de copropietarios, en este caso específico, podemos identificar las personas a las que, en un determinado momento, pertenecen los bienes comunales.

Usando algunas generalizaciones atrevidas, es posible que podamos concluir, sobre la base de estos enunciados, que nuestra proposición sobre la sociedad de responsabilidad limitada pueda reemplazarse por una proposición que sólo contiene los nombres de los socios. En tales circunstancias podríamos claramente ser capaces de escribir.

«los socios A ... A(n) poseen el patrimonio $r »$.

en lugar de hablar sobre la propiedad de la sociedad. De acuerdo con esta línea de pensamiento, presumiblemente se podría también traducir la proposición relativa a la fundación a una proposición que contenga los nombres de algunas personas naturales.

Si se entiende la traducción del «Kennzeichung» a nombres de personas de esta forma, es claro que la interpretación de la sociedad de responsabilidad limitada no está de acuerdo con nuestras leyes en vigor. Después de todo, puede considerarse autoevidente que según la ley finlandesa de Sociedades de responsabilidad limitada no es posible identificar el derecho de propiedad que tiene la compañía con la de sus socios, por ejemplo, sobre el pa- 
trimonio r. Claramente esta identificación es aún menos posible con relación a una fundación. Esto es así porque no es posible indicar las personas físicas de las que, en el sentido normal de la palabra, pueda decirse que poseen la propiedad de la fundación.

\section{3}

¿Se puede, entonces, aun en general, dar un contenido a la posibilidad de traducción tal que se evitaran las dificultades que se han mencionado? En mi opinión, puede hacerse. Apoyándose en ciertas ideas presentadas por Manfred Moritz, se podría sugerir la siguiente respuesta. (Con todo, hay razones muy fuertes para recalcar que la posición de Moritz no puede entenderse de ninguna manera como una interpretación de la aportación de Lahtinen. No me ha sido posible saber si, cuando presentó su opinión, era consciente del punto de partida de Lahtinen).

Moritz toma la siguiente proposición como el objeto de su estudio

«S es un representante de la persona jurídica $\mathrm{JP} »(\ll \mathrm{S}$ ist Stellvertreter der juristischen Person JP»)

y recalca al mismo tiempo que se trata de un concepto unitario («einheitlicher Begriff»). En otras palabras, no puede entenderse como una proposición según la cual $\mathrm{S}$, en el sentido jurídico-técnico usual de la palabra, representa un sujeto. Así, las siguientes expresiones son eliminadas del estudio: «JP es un sujeto de derecho», «A es una persona jurídica» y especialmente la proposición «La persona jurídica JP existe». Es interesante notar que en opinión de Moritz, la cuestión que conduce a tal respuesta está formulada incorrectamente ( $«$ Aus diesem Grunde ist die Frage nach der Existenz der juristischen Person hinfällig. Die Frage ist falsch gestellt»). El problema no está en la clarificación del contenido significativo de la palabra «persona jurídica» sino de la proposición unitaria «S es un representante de la persona jurídica JP».

Si lo he entendido correctamente, las ideas de Moritz son las siguientes. Cuando una sociedad se establece e inscribe, el cambio más importante de hecho (o al menos uno de los cambios más importantes) es que el estatus de ciertas personas, tales como los miembros del consejo de administración o del gerente, cambia. Después de la inscripción se hace referencia, por ejemplo, al gerente, cuando se presenta como tal, con los términos «representante de la sociedad de responsabilidad limitada $X »$. Sin embargo, no se debe trazar un estrecho paralelismo entre esto y, por ejemplo, la autorización «ordinaria» atendiendo sólo a la forma en que se usa el lenguaje. El representante de una persona jurídica tiene una posición especial. Cuando hablamos de una persona jurídica tratamos con un fenómeno único: usando una frase de Moritz, tratamos con derechos y obligaciones «boomerang».

Tomemos un ejemplo para ilustrarlo. Cuando un representante lleva a cabo un acto jurídico, por ejemplo, compra bienes para su representado, una persona física, el acto jurídico obliga inmediatamente al representado. Se transforma en propietario como parte en el contrato.

No obstante, puede pensarse fácilmente en una situación donde el «representante» adquiere una posición más fuerte sobre la base de un contrato. Se le puede autorizar a resolver todos los asuntos en nombre de su represen- 
tado, y tener órdenes para atender todas las obligaciones de su representado. Si es así, entonces llegamos a una situación donde el representado de hecho no tiene nunca que «aparecer». Cuando el representante celebra un contrato, las obligaciones que surgen de este contrato llegan a afectarle como un boomerang. Lo mismo se aplica a los derechos que aumentan sobre la base del contrato. Un ejemplo especialmente típico de esto lo proporciona el Derecho finlandés de la institución de tutela legal. Mientras existe la tutela el menor no participa activamente de ninguna forma en los actos jurídicos. Cualesquiera actos jurídicos en los que participa son inválidos. Los contratos son firmados (y sólo pueden ser firmados) por el tutor en «nombre» del menor.

También, cuando se estudia la representación de una sociedad de responsabilidad limitada y otras personas colectivas, pronto se nota que en muchos aspectos la situación es comparable con la situación mencionada. Cuando un representante, por ejemplo, el gerente o el consejo de administración, actúa en nombre de la sociedad, de acuerdo con las disposiciones vigentes significa que el mismo actor (el gerente o el consejo de administración) tiene el derecho y/o está obligado a realizar ciertos actos. Esto sucede más claramente cuando se trata de la llamada sociedad de un solo hombre, pero la estructura de esta situación no es diferente aunque se trate de una sociedad «ordinaria», una fundación, etc.

Cuando se usa de esta forma, la proposición «S es un representante de la persona jurídica $J P »$, según Moritz, significa que la persona física $S$ en nuestro ejemplo tiene ciertos derechos $(\mathrm{R}, \ldots, \mathrm{R}(\mathrm{n})) \mathrm{y}$, por otra parte, ciertas obligaciones $(\mathrm{P}, \ldots, \mathrm{P}(\mathrm{n}))$. La proposición que Moritz usa como ejemplo $(\mathrm{S}(1))$ puede, en otras palabras, traducirse en una proposición relativamente compleja que hace referencia a los derechos y obligaciones de personas físicas $(\mathrm{S}(2))$. En nombre propio deseo añadir algo. Desde el punto de vista de la lógica de enunciados, la traducción del «Kennzeichung» a nombres de personas significa que la proposición $\mathrm{S}(1)$, que hace referencia a una expresión colectiva tal como «sociedad de responsabilidad limitada», es reemplazada por una conjunción de proposiciones $(S(2))$ que establecen que la persona $S$ pertenece a una determinada categoría en una norma jurídica. Cuando se interpreta de esta forma, nuestro problema se reduce a un estudio de las posibilidades lógicas de reemplazar ciertas complicadas proposiciones por otros enunciados donde la expresión colectiva en cuestión no aparece.

\section{4}

Después de esto, volvamos por un momento a nuestra expresión original «La sociedad de responsabilidad limitada X posee el patrominio $\mathrm{r}$ ». Nos preguntamos lo que se está realmente intentando describir con esta proposición.

Teniendo en cuenta lo que se acaba de decir, la respuesta puede por ejemplo tomar la siguiente forma: sobre la base de ciertos acuerdos y actos (los estatutos de la sociedad, la inscripción, etc.) de (las personas físicas) A... A(n) dentro del ámbito de un cierto sistema de normas, la persona (física) T tiene una posición tal que puede legalmente, como el representante de la sociedad (en nombre de la sociedad), firmar un documento de venta relativa al patri- 
monio. ¿Qué implica esto? Como una consecuencia de la transferencia, ciertas personas que pertenecen a esta organización, tal como $\mathrm{T}$, tienen ciertos derechos y obligaciones. Pueden darse ejemplos que incluyen el derecho a demandar si el derecho de propiedad es violado, y la obligación de tener la propiedad registrada. El acto en nombre de la sociedad, la comparecencia como el representante de la sociedad, crea por ejemplo unos derechos y obligaciones «boomerang» (como las mencionadas) para el mismo actor. Cuando se trata de una sociedad, la propiedad significa así que ciertas personas que pueden ser identificadas sobre la base de ciertos documentos tienen derechos y obligaciones tal como se establecen en el Derecho de sociedades.

No parece que tenga sentido formular la cuestión del derecho de propiedad de la sociedad de otra forma. En otras palabras, es innecesario discutir la cuestión de, por ejemplo, si aun, a pesar de todo, los miembros de la sociedad poseen o no en último término la propiedad de la sociedad. Tal formulación se basa sobre la idea psicológica extraída del uso cotidiano en el que se puede indicar siempre un sujeto en el sentido convencional para todos los derechos. Debería señalarse especialmente que al intentar estudiar la situación cuando la sociedad existe, el resultado que alcanzamos no está afectado de ninguna forma por el hecho de que, a la disolución de la sociedad, los miembros puedan recibir la propiedad que ha «pertenecido» previamente a la sociedad. Es especialmente problemático si, con referencia a esto, se puede decir en general que ya en la disolución de la sociedad los miembros tienen un cierto derecho por ejemplo a la propiedad de la sociedad. En todos los casos parece muy violento llamar a este «derecho» el derecho de propiedad de los miembros. Cuando se trata de una sociedad, la propiedad es también en general un fenómeno tan atípico que, a pesar de la similitud en la terminología, no parece existir ninguna razón para igualar éste con el derecho de propiedad de las personas físicas.

Hasta donde llego a ver, es claro que si se hace referencia específicamente a los aspectos lógicos descritos antes, el problema tradicional de la persona jurídica y las ficciones desaparecen de una forma natural. En tal caso, no se puede dirigir contra este punto de vista la objeción de que no es aplicable a todos los tipos de personas colectivas, tal como las fundaciones. Esto es así porque ya no se constituye un sujeto en cada caso; en su lugar, el problema consiste en traducir una proposición en otra. El significado (el valor de verdad) de las expresiones que contienen términos colectivos («La sociedad de responsabilidad limitada X posee el patrimonio $\mathrm{r} » \mathrm{O}$ «la propiedad de los socios no se puede embargar por la deuda de la sociedad») se muestra en la forma en que discutimos los contenidos de las disposiciones relevantes (tal como las disposiciones de la Ley de responsabilidad limitada). Con referencia a Wittgenstein podemos quizá hablar sobre juegos del lenguaje («Sprachspiel») que se aplican a esta rama del Derecho. Estos juegos nos revelan los contenidos significativos de las proposiciones que estudiamos. Se trata sólo de que usamos el lenguaje de una forma relativamente especial, diferente del lenguaje cotidiano.

(Trad. de José Pedro Úbeda y Juan Ruiz Manero). 


\section{BIBLIOGRAFÍA}

Aarnio, Aulis, Nagra tankar om oskiftat dödsbo som juridisk person. Tidskrift utgiven av Juridiska Föreningen i Finland (JFT), 4/1969 p. 342 ff. Perillisen oikeusasemasta. Porvoo/1967 (German summary).

Prinz, Alois, Pandekten II. Leipzig 1868 p. 979 ff.

Lahtinen, Osvi, Till läran om juridiska persorner. Tidskrift fo Retsvitenskap (TfR) 1949 p. $50 \mathrm{ff}$.

Moritz, Manfred Sind die juristischen Personen Fiktionen. Festskrift för Karl Olivecrona. 1964 p. 442 ff. 